\title{
Radioterapia esplénica: a propósito de 8 casos. Indicaciones y revisión de la literatura
}

\author{
C. IBÁÑEZ VILLOSLADA, C. GONZÁLEZ SAN SEGUNDO, M. V. DE TORRES \\ OLOMBRADA, R. GARCÍA GARCÍA, J. A. SANTOS MIRANDA
}

Servicio de Oncología Radioterápica. Hospital General Universitario Gregorio

Marañón. Madrid

\section{RESUMEN}

Las indicaciones de la radioterapia (RT) esplénica en los desórdenes hematológicos incluye la irradiación del bazo en aquellos procesos linfoproliferativos, que cursan con infiltración del mismo, el tratamiento paliativo de la esplenomegalia en las enfermedades malignas como la leucemia linfoide crónica o los síndromes mieloproliferativos, que cursan con dolor por la distensión capsular del bazo, y las citopenias secundarias al hiperesplenismo.

En este trabajo presentamos la experiencia acumulada en el tratamiento de la esplenomegalia con RT del Hospital General Universitario Gregorio Marañón en los últimos 5 años, analizando sus indicaciones, resultados y toxicidad así como una revisión actualizada de la literatura.

PALABRAS CLAVE: Radioterapia. Esplenomegalia.
SPLENIC IRRADIATION: A PROPOSAL OF 8 CASES. CLINICAL INDICATIONS AND LITERATURE REVIEW

\begin{abstract}
Clinical indications of splenic irradiation in haematological disorders include the irradiation in lymphoproliferative disorders with spleen infiltration, palliative treatment of splenomegaly in malignant diseases like chronic lymphocytic leukaemia or myeloproliferative disorders, with the purpose of relief from abdominal pain associated with capsular enlargement size and decrease cytopenias secundaries to hypersplenism.

This paper reports our experience with spleen irradiation in the Hospital General Universitario Gregorio Marañon in the last five years, and analizes indications, results and toxicity, and an actual review of the literature.
\end{abstract}

KEY WORDS: Radiotherapy. Splenomegaly.

Ibáñez Villoslada C, González San Segundo C, de Torres Olombrada MV, García García R, Santos Miranda JA. Radioterapia esplénica: a propósito de 8 casos. Indicaciones y revisión de la literatura. An Med Interna (Madrid) 2007; 24: 231-234.

\section{INTRODUCCIÓN}

La esplenomegalia es un signo característico de determinadas patologías hematológicas como algunos síndromes mielodisplásicos y los síndromes mieloproliferativos crónicos. Sus principales consecuencias son el dolor, por la distensión capsular y la tracción sobre los ligamentos suspensorios del bazo, el riesgo de desarrollar un infarto esplénico y la aparición de pancitopenia en relación con la presencia de hematopoyesis extramedular o por la infiltración leucémica del bazo $(1,2)$.

En los casos que presentan esplenomegalia sintomática, descartada la realización de esplenectomía, la irradiación esplénica es una buena opción terapéutica ya que consigue una alta tasa de respuestas tanto en la disminución del tamaño del bazo como en la mejoría del dolor abdominal $(3,4)$. Sin embargo, se conoce muy poco sobre el mecanismo exacto de actuación de la irradiación esplénica y su repercusión en la evolución de la enfermedad hematológica y en los recuentos celulares (3).
En el presente estudio exponemos la experiencia del Hospital General Universitario Gregorio Marañón en el tratamiento de la esplenomegalia con RT con la descripción de 8 casos tratados en los últimos 5 años. Comparamos nuestros resultados con los publicados en la literatura con el fin de discernir la rentabilidad terapéutica de la RT esplénica, la dosis a recomendar, la posibilidad de reirradiación y la toxicidad radioinducida de este procedimiento.

\section{CASOS APORTADOS}

Desde enero de 2002 hasta enero de 2006, 8 pacientes con esplenomegalia sintomática han sido tratados con irradiación esplénica en nuestro servicio. Las características principales de los pacientes se resumen en la tabla I. Cinco pacientes estaban diagnosticados de linfoma marginal esplénico, dos de leucemia linfoide crónica y uno de leucemia mielo-monocítica juvenil. El rango de edad estaba comprendido entre los 3 y los 81 años. En 5 pacientes la RT se administró

Trabajo aceptado: 18 de diciembre de 2006

Correspondencia: Carmen Ibáñez Villoslada. Servicio de Oncología Radioterápica. Hospital General Universitario Gregorio Marañón. C/ Doctor Esquerdo, 46. 28007 Madrid. e-mail: carmelaiv@yahoo.es 
TABLA I

\begin{tabular}{|c|c|c|c|c|c|c|c|c|}
\hline \multicolumn{9}{|c|}{ CARACTERÍSTICAS DE LOS PACIENTES } \\
\hline Patología & $N$ & Sexo & Edad & Dosis (Gy) & Dosis/Frac & Trasf. & Tto. Previo & Observaciones \\
\hline $\begin{array}{l}\text { Linfoma marginal } \\
\text { esplénico }\end{array}$ & 5 & $\begin{array}{l}V \\
M \\
V \\
M \\
V\end{array}$ & $\begin{array}{l}55 \\
74 \\
81 \\
77 \\
79\end{array}$ & $\begin{array}{l}12 \\
10 \\
1 * \\
6,3^{* *} \\
10\end{array}$ & $\begin{array}{c}1,8 \\
2 \\
1 \\
0,9 \\
1\end{array}$ & $\begin{array}{l}\text { No } \\
\text { No } \\
\text { No } \\
\text { No } \\
\text { No }\end{array}$ & $\begin{array}{l}\text { Sí } \\
\text { No } \\
\text { No } \\
\text { No } \\
\text { No }\end{array}$ & $\begin{array}{l}\text { * Recibió una única sesión por } \\
\text { importantes co-morbilidades } \\
\text { * No finalizó por severa neutro- } \\
\text { penia y deterioro de estado } \\
\text { general }\end{array}$ \\
\hline $\begin{array}{l}\text { Leucemia linfoide } \\
\text { crónica }\end{array}$ & 2 & $\begin{array}{l}M \\
V\end{array}$ & $\begin{array}{l}69 \\
76\end{array}$ & $\begin{array}{l}12 \\
20\end{array}$ & $\begin{array}{c}0,8 \\
1\end{array}$ & $\begin{array}{l}\text { No } \\
\text { No }\end{array}$ & $\begin{array}{l}\text { Sí } \\
\text { No }\end{array}$ & \\
\hline $\begin{array}{l}\text { Leucemia mielo- } \\
\text { monocítica juvenil }\end{array}$ & 1 & V & 3 & 5 & 0,5 & Sí & Sí & $\begin{array}{l}\text { Reirradiación un mes después } \\
\text { por crecimiento esplénico: dosis } \\
\text { total } 10 \mathrm{~Gy}\end{array}$ \\
\hline
\end{tabular}

N: número de pacientes; Trasf.: requerimientos transfusionales durante la radioterapia; Gy: gray; V: varón; M: mujer; Frac: fracción.

como único tratamiento y en 3 tras el fracaso a la quimioterapia (QT). En 7 pacientes se descartó la esplenectomía por la comorbilidad asociada o por el rechazo del paciente a la cirugía. En 3 pacientes se describían datos hematológicos asumibles como secundarios a un hiperesplenismo.

Respecto al tratamiento con RT, en todos los pacientes se realizó la planificación mediante 2 campos opuestos anteroposterior y posteroanterior (AP-PA), con tamaños que oscilaron desde $14 \times 17 \mathrm{~cm}^{2}$ hasta $25 \times 16 \mathrm{~cm}^{2}$. En función del espesor del paciente se emplearon fotones X de 6 ó $15 \mathrm{MV}$. El niño diagnosticado de leucemia mielomonocítica juvenil necesitó soporte anestésico en todas las sesiones. Fue el único paciente que fue reirradiado, sin objetivarse una mejoría clínica con la segunda irradiación.

El resultado y la tolerancia al tratamiento fue buena en todos los casos salvo en dos. En el primero, el paciente recibió una única sesión por presentar un cuadro de insuficiencia respiratoria que precisó ingreso hospitalario (diagnosticado de EPOC severo clase funcional III-IV/IV con oxígeno domiciliario). En el segundo, tras alcanzar una dosis de 6,3 Gy, hubo que suspender el tratamiento por presentar neutropenia severa y deterioro del estado general. En 6 de los 8 pacientes tratados se objetivó una mejoría clínica con alivio del dolor secundario a la reducción del tamaño del bazo. En todos ellos las necesidades transfusionales disminuyeron en los meses posteriores a la irradiación. El tiempo medio de supervivencia tras la RT fue de 18,7 meses. Cinco de los 8 pacientes siguen vivos con un seguimiento superior a los 2 años.

\section{DISCUSIÓN}

El inicio del empleo de la RT para el tratamiento de la esplenomegalia sintomática se remonta al año 1903 (5) y, hasta la fecha, su mecanismo de actuación no ha sido suficientemente aclarado (3). El conocimiento de la fisiopatología del bazo revela su participación en funciones inmunológicas, de "aclaramiento" de elementos formes opsonizados que condiciona la fagocitosis de hematíes envejecidos o anómalos, sin olvidar su papel como órgano de reserva hematopoyética (el bazo acumula el $30 \%$ de las plaquetas circulantes), así como la función de almacén de hierro en forma de hemosiderina y ferritina y, por último, como foco potencial de hematopoyesis extramedular en situaciones especiales $(1,2)$.

En general, la RT esplénica se ha indicado en el tratamiento de la esplenomegalia por infiltración leucémica en los síndromes linfoproliferativos crónicos, como la leucemia linfoi- de crónica (LLC) o la leucemia prolinfocítica (LPL), y en los síndromes mieloproliferativos crónicos (SMCs), sobre todo en la metaplasia mieloide y en la leucemia mieloide crónica (LMC). En estos últimos, la esplenomegalia es secundaria a la hematopoyesis extramedular condicionada por la mielofibrosis típica de estos desórdenes hematológicos $(3,4,6)$. La púrpura trombocitopénica idiopática, asociada o no al VIH, completan las principales indicaciones de la irradiación en la esplenomegalia $(3,7)$.

Aunque el objetivo principal de la RT esplénica es local, paliando los síntomas derivados de la esplenomegalia (dolor, síndrome constitucional...), la irradiación puede además disminuir la actividad del sistema reticuloendotelial del bazo e incluso, como explican Weinmann et al, tener un efecto sistémico responsable en parte de la pancitopenia y de la desaparición de las adenopatías localizadas a distancia de la región esplénica (4). Para explicar este efecto sistémico se barajan distintos mecanismos: muerte celular por efecto directo de la radiación, cambios en las subpoblaciones linfocitarias producidos por inmunomodulación, cambios en el patrón de secreción de citoquinas y, a dosis mayores de 20 Gy, la anulación funcional del bazo con las mismas consecuencias que la esplenectomía $(3,4)$.

Como ocurre en nuestra serie, en la literatura consultada las esplenomegalias causadas por las LLCs, los linfomas esplénicos de la zona marginal y los SMCs son las indicaciones más frecuentes de tratamiento radioterápico. La respuesta a la RT se evalúa no sólo por la disminución del tamaño del bazo, sino también por el alivio clínico del dolor y la disminución de los requerimientos transfusionales (8). La Tabla II recoge la experiencia publicada en series con más de 10 pacientes. En general, la reducción del tamaño del bazo ocurre en el $80-100 \%$ de los pacientes con SMCs y en el 50-63\% de las LLCs. La duración media de la respuesta es de 6 meses. Respecto a la clínica dolorosa, la mejoría se consigue en el $62-100 \%$ de los SMCs y en el 60-75\% de las LLCs, con una duración media de 7 meses $(3,4,6)$. En los pacientes con mielofibrosis se ha objetivado hasta un $50 \%$ de descenso en las necesidades transfusionales (8).

Respecto a la técnica de irradiación, lo habitual es la utilización de un campo directo AP o dos campos opuestos AP$\mathrm{PA}$ con fotones $\mathrm{X}$ de energía ascendente en relación directa con el aumento de espesor del paciente (4-18 MV). No existe 
TABLA II

SERIES DE IRRADIACIÓN ESPLÉNICA

\begin{tabular}{|c|c|c|c|c|c|c|c|}
\hline & N & Patología & Indicación & Dosis total (Gy) & Fraccionamiento & Energía & Resultados \\
\hline McFarland (3) & 17 & $\begin{array}{c}\text { LLC, LMC, } \\
\text { LMA, PTI } \\
\text { MMM }\end{array}$ & $\begin{array}{c}16 \text { pacientes } \\
\text { esplenomegalia } \\
1 \text { paciente PTI }\end{array}$ & $3,5-6$ & $\begin{array}{c}1^{a} \text { sem: } 2 \text { fr } 0,5 G y \\
2^{\text {a sem: }} 2 \text { fr 0,75 Gy } \\
3^{a} \text { sem: } 2 \text { fr } 1 \text { Gy }\end{array}$ & $6-18 \mathrm{MV}$ & Mejoría clínica \\
\hline Elliot (6) & 23 & $M M M$ & $\begin{array}{c}\text { Esplenomegalia } \\
\text { Dolor } \\
\uparrow \text { Rápido tamaño } \\
\uparrow \text { Volumen plasma }\end{array}$ & $3-13,6$ & 2-17 fracciones & $M E$ & $\begin{array}{c}\downarrow \text { Citopenia } \\
\downarrow \text { esplenomegalia }\end{array}$ \\
\hline Blauth (7) & 10 & PTI en VIH & $\begin{array}{l}\text { Dolor abdominal } \\
\text { Sangrado } \\
\text { espontáneo } \\
\text { Trombocitopenia } \\
\text { persistente }\end{array}$ & $5-10$ & $1 \mathrm{~Gy} / \mathrm{fr}$ & $6-18 \mathrm{MV}$ & $\begin{array}{c}\geq 50 \% \text { p plaquetas } \\
75 \% \text { mejoría } \\
\text { sintomática }\end{array}$ \\
\hline
\end{tabular}

N: número de pacientes; Gy: gray; LLC: leucemia linfoide crónica; LMC: leucemia mieloide crónica; LMA: leucemia mieloide aguda; PTI: púrpura trombocitopénica idiopática; MMM: mielofibrosis metaplasia mieloide; sem: semana; fr: fracción; MV: megavoltios; ne: no especificado; VIH: virus de la inmunodeficiencia humana.

consenso con respecto al fraccionamiento ni a la dosis total necesaria. Los esquemas más habituales emplean 5 fracciones/semana, 0,5-1 Gy/fracción, hasta alcanzar una dosis total de 5-10 Gy. Dada la exigua literatura al respecto, es difícil obtener unas conclusiones consistentes, aunque se ha sugerido que dosis totales menores de 3-5 Gy pueden ser insuficientes y que mayores de 10 Gy pueden no aportar beneficio alguno. Parece que en los casos de mielofibrosis se recomiendan dosis totales más bajas, entre 3 y 5 Gy, en 5 fracciones/semana de 0,1-0,5 Gy/fracción, y que en los casos de LLC, linfoma esplénico de la zona marginal LPL o leucemia de células peludas, la dosis total de $10 \mathrm{~Gy}$, en 5 fracciones/semana de 0,1-0,5 Gy/fracción, puede considerarse el estándar. McFarland et al y Elliot et al defienden los tratamientos intermitentes con 1-3 fracciones a la semana; en su experiencia, consiguen el mismo porcentaje de respuestas y valoran con más exactitud cómo se desarrolla la pancitopenia entre las sesiones $(3,4,6,9)$.

A lo largo del tratamiento es preciso hacer una valoración diaria del tamaño del bazo ya que, en función de la respuesta clínica, se deben ir reduciendo las dimensiones del campo con el fin de reducir la toxicidad. Además, se requiere un control hematológico exhaustivo ya que las posibles consecuencias a este nivel son variadas e impredecibles; la anemia y la trombopenia, en ocasiones, son de etiología multifactorial y pueden ser el resultado tanto de la infiltración tumoral de la médula ósea como del aumento de la degradación celular en el bazo y de la aparición de fenómenos autoinmunes $(4,6)$. Debido a ello, los efectos a nivel sanguíneo dependerán de cuál de estos mecanismos predomine en cada patología concreta. En aquellos casos en los que la citopenia se deba a la degradación celular esplénica, la disminución del tamaño del bazo mejorará dicha citopenia. Sin embargo, en aquellos en los que el origen de la esplenomegalia sea la hematopoyesis extramedular por insuficiencia de la médula ósea, la irradiación esplénica puede incluso agravarla $(4,10)$.

Respecto a la conveniencia de realizar una TAC de planificación, algunos autores la recomiendan en aquellos pacientes con patología renal conocida y en los casos en que se prevea la posibilidad de una reirradiación. Aunque las dosis administradas habitualmente no superan la dosis de toxicidad limitante de la función renal, en algunos pacientes que han recibido varias tandas de irradiación se ha llegado a alcanzar dosis totales superiores a los $25 \mathrm{~Gy}$, teóricamente por encima de la dosis limitante, si bien resulta difícil calcular la dosis biológica equivalente recibida por el riñón izquierdo debido al tipo de fraccionamiento (hipofraccionamiento) y al tiempo transcurrido entre las irradiaciones (decay) $(4,9)$.

La reirradiación en los casos de progresión clínica o sintomática es una práctica habitual, bien tolerada y con una toxicidad aceptable $(3,4,6,8,9)$; además no disminuye ni el índice de respuestas ni la duración de la mismas. En algunas series de pacientes con mielofibrosis se ha llegado a reirradiar al $66 \%$ de los enfermos $(3,9)$.

La tolerancia aguda al tratamiento es buena, siendo los efectos secundarios más frecuentes las náuseas, los vómitos y la astenia, generalmente sin repercusión importante para el paciente. En cuanto a la toxicidad hematológica, ésta puede variar desde una citopenia asintomática hasta una disminución severa de las cifras sanguíneas que requiera transfusiones y obligue incluso a suspender el tratamiento. En general, la trombocitopenia no es una contraindicación para la irradiación y los motivos para suspenderla vienen condicionados por el riesgo de sepsis o de hemorragia y por el estado funcional del paciente. Las complicaciones hematológicas fatales y la sepsis suelen ser las principales causas de muerte en los pacientes irradiados, con cifras cercanas al 13\% (3,6,8-10).

Mención aparte merece la esplenomegalia en pacientes con VIH, en los que una de las causas más frecuente es la trombocitopenia autoinmune (7). Aunque el mecanismo que la produce aún no se conoce con exactitud, se ha identificado la producción de auto-anticuerpos plaquetarios como uno de los principales factores implicados. La indicación de la RT en estos casos, además de la puramente sintomática, se fundamenta en el beneficio sobre el recuento plaquetario, al tratar el foco principal de producción de anticuerpos y de posterior destrucción de las células opsonizadas. Con dosis totales bajas, entre 5 y $10 \mathrm{~Gy}$, se consigue una tasa de respuestas del $90 \%$ de forma transitoria y de un $10-20 \%$ mantenidas en el 


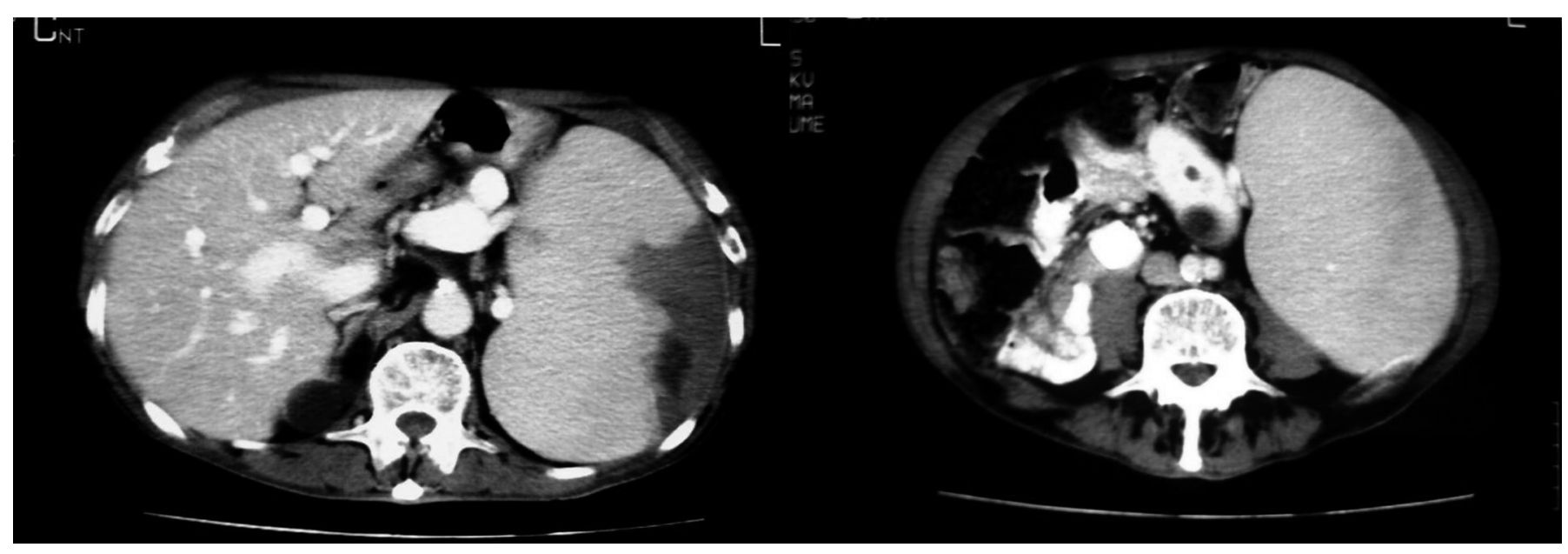

Fig. 1. Imágenes de esplenomegalia en paciente con linfoma marginal esplénico.

tiempo, por lo que a pesar de no ser unos resultados comparables con los de la cirugía, debe considerarse como una alternativa de tratamiento.

Por último, estos pacientes alcanzan supervivencias medias de 3 a 5 años, desde el diagnóstico, y el papel que puede jugar la RT es dificil de cuantificar (4). En la actualidad, la mayor parte de los pacientes han sido sometidos a tratamiento quimioterápico con fármacos como la hidroxiurea, la fludarabina o el imatinib. La indicación de la RT tiene lugar cuando el paciente deja de responder a la QT o cuando hay progresión del dolor, de la anemia o de la trombopenia. En los SMCs, la mediana de supervivencia tras la irradiación es de 22 meses, pero esta cifra está condicionada por otros factores como la existencia de conversión leucémica, los requerimientos trans- fusionales, la respuesta previa a la QT y, sobre todo, el estado general del paciente (6).

En definitiva, la RT como terapia paliativa en pacientes con esplenomegalia es una alternativa eficaz a la esplenectomía, no sólo como alivio del dolor sino también como tratamiento de las citopenias con descripción, en algunas series, de respuesta tumoral. Su morbilidad y mortalidad son comparables a las de la cirugía, considerando además que los pacientes que se irradian son aquéllos con peor estado general. El conocimiento de la fisiopatología del bazo y de la causa de la esplenomegalia ayudan a comprender cuál es el mecanismo de acción de la RT y a individualizar mejor la prescripción y el objetivo del tratamiento.

\section{Bibliografía}

1. Tomás JF, Moraleda JM. El bazo. Esplenomegalias. Hiperesplenismo. En: Moraleda JM, ed. Hematología. Madrid: Ed Luzán 2ª ed; 1996. p. 377-86.

2. Castro del Pozo S. Fisiopatología de los ganglios linfáticos y del bazo. En: Castro del Pozo S ed. Manual de Patología General. Barcelona: Masson, S.A. $5^{\mathrm{a}}$ ed; 1993. p. 345-50.

3. McFarland JT, Kuzma C. Palliative irradiation of the spleen. Am J Clin Oncol 2003; 26: 178-83.

4. Weinmann M, Becker G, Einsele H, Bamberg M. Clinical indications and biological mechanisms of splenic irradiation in chronic leukaemias and myeloproliferative disorders. Radiother Oncol 2001; 58: 235-46.

5. Senn N. Case of splenomedullary leukaemia successfully treated by the use of roentgen ray. Med Record 1903; 63: 281.

6. Elliott MA, Chen MG, Silverstein MN, Tefferi A. Splenic irradiation or symptomatic splenomegaly associated with myelofibrosis with myeloid metaplasia. Br J Haematol 1998; 103: 505-11.

7. Blauth J, Fisher S, Henry D, Nichini F. The role of splenic irradiation in treating HIV-associated inmune thrombocytopenia. Int J Radiat Oncol Biol Phys 1999; 45: 457-60.

8. Bouadballah R, Coso D, Gonzague-Casabianca L, Alzieu C, Resbeut M, Gastaut JA. Safety and efficacy of splenic irradiation in the treatment of patients with idiopatic myelofibrosis: A report on 15 patients. Leuk Res 2000; 24: 491-5.

9. Jyothirmayi R, Coltart S. An audit of the indications for and techniques of palliative splenic radiotherapy in the UK. Clin Oncol 2005;17:192-4.

10. Belka C, Ottinger H, Kreuzfelder E, et al. Impact of localized radiotherapy on blood immune cells counts and function in humans. Radiother Oncol 1999; 50: 199-204. 